\title{
Varicella zoster virus DNA in throat swabs
}

\author{
T Ozaki, H Miwata, Y Matsui, S Kido, K Yamanishi
}

\begin{abstract}
Eighty one throat swabs from 18 patients infected with varicella were tested for varicella zoster virus DNA by the polymerase chain reaction. The positive rates were $26 \cdot 2 \%$ (11/42) during the incubation period and 89.7\% (35/39) after clinical onset. The results indicate the pharynx is a site for early replication of the virus.
\end{abstract}

Varicella, caused by primary infection with varicella zoster virus, is a common and highly contagious disease of childhood. The airway of patients infected with varicella is considered to be the main source of infection during the end of the incubation period and the first days of the rash. Isolation of the virus from the pharynx is usually difficult, however, and in a previous study we reported five isolates of the virus $(4 \cdot 3 \%)$ from 117 children with varicella by using human embryonic lung cell cultures. ${ }^{1}$

The polymerase chain reaction is a new technique that amplifies a specific sequence of DNA efficiently and is used for the detection of viral DNA in clinical specimens. Recently we established a method for detecting varicella zoster virus DNA by the polymerase chain reaction. ${ }^{2}$ We report the results of our attempts to detect viral DNA in the pharynx of normal children with varicella infection by this technique.

\section{Patients and methods}

This study was conducted at the paediatric outpatient clinic of Showa Hospital over a six month period in 1989 . When a child presented with varicella any siblings who had no history of varicella infection were asked to visit the clinic, and throat swabs were taken during the incubation period and after clinical onset of the infection. Eighteen normal siblings of children with varicella, who had no known underlying diseases and were not receiving immunosuppressive treatment, were included in this study; their ages ranged from 4 months to 8 years. The study was also performed on 16 healthy children as negative controls; their ages ranged from 5 months to 14 years of age. After the project was explained consent was obtained from the parents.

A total of 81 throat swabs were obtained from the 18 siblings of children with varicella. Forty two swabs were taken during the incubation period and 39 after appearance of the rash. Only one throat swab was taken from each of the 16 healthy control children. All swabs were placed in $3 \mathrm{ml}$ of phosphate buffered saline and stored at $-80^{\circ} \mathrm{C}$ before analysis.

The method used for detection of the viral DNA by the polymerase chain reaction has been described previously. ${ }^{2}$ Four oligonucleotide pairs of 25 nucleotides (regions of thymidine kinase, thymidylate synthetase, glycoprotein I, and immediate early gene) were synthesised. The first three oligonucleotide pairs could be used as primers on the basis of specific DNA amplification. The amplification reaction was carried out in a DNA thermal cycler (PerkinElmer Cetus). The amplified product was detected by direct gel electrophoresis with ethidium bromide staining and by Southern blot hybridisation with a homologous ${ }^{32} \mathrm{P}$-labelled cloned DNA probe (fig 1).

For determination of the sensitivity of the polymerase chain reaction in this system, a sample of cloned varicella zoster virus DNA (glycoprotein I region) was mixed with human placental DNA and this DNA was amplified with primers of glycoprotein I. The fragments of varicella zoster virus DNA with the same molecular weight as the expected DNA could be detected in agarose gel by ethidium bromide staining at a level of $5 \times 10^{3}$ copies, and 50 copies of the viral DNA could be detected by Southern blot hybridisation assay.

\section{Results}

Figure 2 shows the age and sex of the 18 children with varicella, maximum body temperature during the disease, and the results of the analysis by the polymerase chain reaction in throat swabs according to time of appearance of disease. In 11 of $42(26.2 \%)$ throat swabs taken during the incubation period, varicella zoster virus DNA could be detected by the polymerase chain reaction with Southern blot hybridisation. We detected viral DNA in one swab 15 days before onset of the disease and in four of nine $(44 \cdot 4 \%)$ swabs during the incubation period

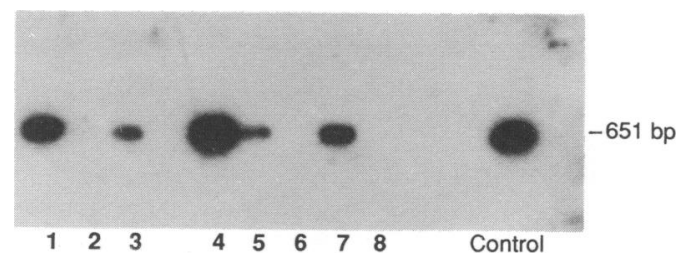

Figure 1 Autoradiographafter Southern blot hybridisation with ${ }^{32} P$-labelled probe for eight pharyngeal swabs and $a$ positive control. Five samples $(1,3,4,5$, and 7) contain varicella zoster virus $D N A, b p=$ base pairs. 


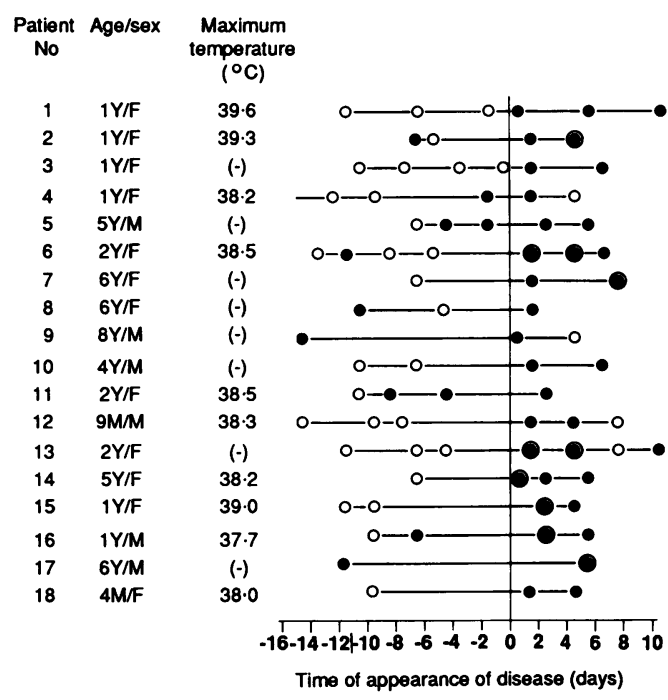

Figure 2 The results of the analysis by polymerase chain reaction in throat swabs according to time of appearance of disease. Not detected (open circle), detected (closed circle), and detected by direct gel electrophoresis (large open circle). Body temperature (-) was $<37.5^{\circ} \mathrm{C}$ during the disease; $Y=$ years, $M=$ months.

within five days before the onset. The viral DNA could be detected in 35 of $39(89.7 \%)$ throat swabs after appearance of the rash. The detection rate during the first three days after clinical onset of disease was $100 \%$ by the polymerase chain reaction with Southern blot hybridisation.

In 10 of $39(25.6 \%)$ swabs after onset of disease, varicella zoster virus DNA was detected not only by Southern blot hybridisation but also by direct gel electrophoresis. On the other hand, no viral DNA could be detected in the swabs during the incubation period by the polymerase chain reaction with direct gel electrophoresis. In the throat swabs of the 16 healthy children no viral DNA was amplified specifically.

\section{Discussion}

In our previous studies we provided evidence for a mononuclear leucocyte associated viraemia occurring routinely during the incubation period and first few days of disease in nearly all normal children who had typical clinical manifestations of varicella. ${ }^{3-6}$ However, isolation of varicella zoster virus from the pharyngeal area is usually difficult in normal children with varicella. It has been reported in a small number of cases after the onset and in no case during the incubation period by using the cell culture technique. ${ }^{1}$

The sensitive method that we established for detection of varicella zoster virus detected viral DNA between 15 days before and 11 days after appearance of the rash in normal children infected with varicella. The rate of detection during the incubation period was $25.6 \%$ (11/ 42), and within five days before onset of disease was $44 \cdot 4 \%$ (4/9). After clinical onset of disease viral DNA could still be detected during the convalescent phase in two patients (1 and 13) and the rate of detection increased to $100 \%$ during the first three days of the rash. The findings were variable in four patients $(2,6,8$, and 13$)$. We think that the difficulty in taking throat swabs from each child in a standardised fashion led to false negatives in a succession of positive findings. The rate of detection did not correlate with the maximum body temperature during the disease.

In a total of 10 swabs varicella zoster virus DNA was detected by direct gel electrophoresis, but these were swabs that were taken after clinical onset of the disease. The sensitivity of Southern blot hybridisation is higher than that by direct gel electrophoresis with ethidium bromide staining. From this point of view more viral DNA (in quantity) was found after clinical onset than during the incubation period.

The primary site of infection and replication of varicella zoster virus has been considered to be mucosa of the upper respiratory tract and/or conjunctivae. These results suggest that the pharynx is a site for early replication of the virus. Despite the fact that no virus could be isolated from the pharyx by cell culture technique, transmission of varicella zoster virus has been reported one to two days before onset of the rash. The results of this study might resolve the apparent paradox. By the use of the polymerase chain reaction we will be able to study further the pathology of varicella zoster virus infection.

1 Ozaki T, Matsui Y, Asano Y, Okuno T, Yamanishi K, Takahashi M. Study of virus isolation from pharyngea 1448-50.

2 Kido S, Ozaki T, Asada $\mathrm{H}$, et al. Detection of varicella-zoster virus (VZV) DNA in clinical samples from patients with VZV by the polymerase chain reaction (PCR). I Clin Microbiol (in press)

3 Ozaki T, Ichikawa T, Matsui Y, et al. Viremic phase in nonimmunocompromised children with varicella. $\mathcal{J}$ Pediat 1984;104:85-7.

4 Asano Y, Itakura N, Hiroishi Y, et al. Viremia is present in incubation period in nonimmunocompromised children with varicella. F Pediatr 1985;106:69-71.

5 Ozaki T, Ichikawa T, Matsui Y, et al. Lymphocyte-associated viremia in varicella. F Med Virol 1986;19:249-53.

6 Asano Y, Itakura N, Hirose S, et al. Viral replication and immunologic responses in children naturally infected with varicella-zoster virus and in varicella vaccine recipients. $\mathcal{F}$ Infect Dis 1985;152:863-8.

7 Trlifajova J, Bryndova D, Ryc M. Isolation of varicella-zoster virus from pharyngeal and nasal swabs in varicella patients. f Hyg Epidemiol Microbiol Immunol 1984;28:201-6. 